\title{
Degradation of fibreglass composites under natural weathering conditions
}

\begin{abstract}
In this study, sets of glass fibre reinforced polymer (GFRP) samples were manufactured and exposed to a semi-arid climate, in Kelowna, B.C., with dry, sunny summers, cold, cloudy winters and all four seasons. The open mould wet lay-up process was used to fabricate both the exposure and control laminates. Different fibre architectures, coating types, and degrees of initial cure were chosen among the study factors via a design of experiments (DOE) approach. Each type of sample was tested for its flexural bending strength, hardness, surface roughness, and specific gravity, before and after a nine month exposure period. Results showed changes in the significance level of different initial laminate design parameters on the mechanical and physical properties of the GFRPs, depending on the indoor (room) or outdoor environment of the parts.
\end{abstract}

Keywords: fiber glass composites, long term performance, natural environmental degradation
Volume I Issue I - 2017

Heinrick M, Crawford B, Milani AS
University of British Columbia, Canada

Correspondence: Milani AS, Composites Research NetworkOkanagan Laboratory, School of Engineering, University of British Columbia, Kelowna, Canada, Email abbas.milani@ubc.ca

Received: March 29, 2017 | Published: April 07, 2017

\section{Introduction}

Long term durability of composites is important to consider when making material selection and processing decisions to manufacture structures. ${ }^{1-5}$ For example, the choice of resin system used for GFRPs has a direct correlation to the durability and lifetime of structures, ${ }^{2}$ where the use of vinyl ester resins lend a greater lifespan, although being more expensive as a raw material, while polyesters are a cheaper, less durable alternative and are more susceptible to degradation by the ingress of water and exposure to ultraviolet radiation. ${ }^{5}$ Similarly, the choice of fibre perform has an impact on the durability of structures. It has been shown that through laboratory-scale tests and modeling approaches, fibre architecture and orientation affect the ingress of water into polymer matrix composites. ${ }^{4}$ Through the mechanism of capillary action, continuous fibres would carry moisture more readily into a laminate in plane. Accelerated aging tests have supported these hypotheses, in addition to showing chemical degradation of polymers by ultraviolet exposure. Other studies have shown that there are correlations between types of environmental degradation and physical or mechanical properties of laminates. ${ }^{1}$ These include reductions in maximum flexural stress of GFRPs, modulus of elasticity, and strain to failure. These correlations, however, are built upon laboratoryscale testing with accelerated exposure, which may not truly reflect the nature of material's "natural" degradation outdoors.

The present study seeks to fill the above gap on natural aging of GFRPs, affecting the laminate mechanical and physical properties such as maximum flexural strength, hardness, surface roughness (surface finish) and specific gravity (density). A set of different GFRPs with different fiber architectures, different initial degrees of cure, and different coating conditions, have been tested using a natural weathering station over a nine-month period. Statistical results from this preliminary study are part a long-term research program with the ultimate goal of generating a set of best-practice guidelines for lifecycle management of GFRPs.

\section{Experiment}

\section{Material preparation}

Material samples used in experiments were fabricated with three major variations in their composition and processing. The three factors include the fibre preform architecture (E-glass plain woven $400 \mathrm{~g} / \mathrm{m}^{2}$ and E-glass random chopped fibre mat $800 \mathrm{~g} / \mathrm{m}^{2}$ ), coating material (Polycor ${ }^{\circledR}$ isophthalic white gel coat or a plain surface), as well as degree of cure (partially and fully cured). All laminates were manufactured using EnvirezTM unsaturated polyester resin with a 1.25 percent by weight methyl ethyl ketone peroxide initiator, at a fibre volume fraction of approximately $35 \%$ for the random chopped mat laminate and $40 \%$ for the continuous plain weave laminates, respectively. Samples were fabricated on a flat sheet of stainless steel 304 alloy as the tooling, for two different time periods, either cured in a controlled ambient environment or cured in an industrial oven. Ambient cured samples were exposed to a controlled environment of $21^{\circ} \mathrm{C}$ and $33 \%$ relative humidity for approximately one day, whereas the fully cured samples were placed in an industrial oven at $50^{\circ} \mathrm{C}$ for a minimum of 6 hours. It was estimated, through the use of cure modeling software RAVEN, that the degree of cure of the cooler samples were approximately 0.84 and that of the ovencured samples were 0.96. Small differences in the degree of cure also existed between the samples of two different fibre architectures, yet these cure differences were smaller than those due to different processing temperatures. After the cure time, samples were cut using an abrasive water jet cutting Omega 2652 Jet Machining Centre. The sample dimensions were in accordance with 3-point-bend (3PB) ASTM standard $(13 \mathrm{~mm} \times 215 \mathrm{~mm})$. Prior to exposing the samples to the exterior environment, the edges were sealed using an acrylic latexbased resin in order to prevent immediate ingress of moisture into the laminates, hence better simulating the exposure of a large, flat sheet of composite in practice. ${ }^{3}$

\section{Experimental methods}

After sample fabrication and curing, some samples were immediately tested for surface roughness, surface hardness, flexural stress and specific density. These tests took place in a controlled laboratory environment at $21^{\circ} \mathrm{C}$ and 33 percent relative humidity. A second control group of samples were established and kept in these controlled laboratory conditions over the nine-month period, while the rest of sample groups were installed on a weathering station at the University of British Columba, for 270 days. After this period, 
the exposed samples were tested in the laboratory for the same type of tests described earlier. The equipment used during testing as well as the testing procedures are outlined in the sub-sections to follow.

Weathering station/Environmental exposure: As pictured in Figure 1 , the weathering station is positioned on an unobstructed roof system of a building exposed to all environmental elements in Kelowna, BC. This station is composed of an aluminum sample exposure rack designed to adjust and accommodate various size specimen. Samples were installed on the exposure rack and the sample ends were manually secured in place by ultra high molecular weight polyethylene clamps, to reduce contamination from the structure. The Campbell Scientific ${ }^{8}$ weathering station was equipped with a CR800 data logger and numerous sensors, including relative humidity and air temperature probe, broadband UV radiometer and TE525 tipping bucket rain gauge. All fabricated samples used for outdoors were installed in the weathering station with flat-mould side up and inclined at a 45 degree angle, in order to resist any potential pooling of water. The rack was oriented to maximize exposure to sunlight throughout the testing period. The samples were installed in February 2014, beginning exposure during winter conditions.

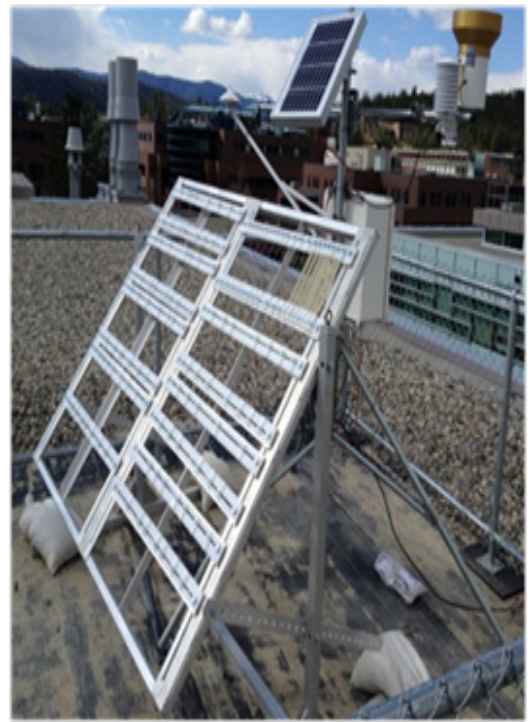

Figure I Weathering station set-up used during the environmental performance evaluation of the GFRP samples.

Roughness tests: The average mean roughness $\left(R_{\mathrm{a}}\right)$ of each sample was measured using a portable Qualitest ${ }^{\mathrm{TM}}$ TR110 roughness tester. An average of ten separate roughness points was taken randomly over the surface midsection of each specimen. Measurements were acquired from the flat-mould side in accordance with the TR110 operating procedures.

Hardness tests: The Shore D hardness of each specimen was measured using a stationary Qualitest ${ }^{\mathrm{TM}}$ HPE-II durometer in accordance to ASTM D2240-05. An average of three random measurements was calculated per specimen along its midsection. Measurements were acquired from the flat-mould side of the specimens.

Flexural tests: Three point bending tests were conducted using an Instron 5969 Dual Column testing machine in accordance to ASTM D7264/D7264M-07. Two repeats were performed with a crosshead speed of $10 \mathrm{~mm} /$ minute and a preloading of $35 \mathrm{~N}$. The preload was found to be effective in stabilising the samples, which was likely due to the high degree of surface variability on the open-moulded side of the laminate. All samples were placed flat mould side upwards during three-point bending tests (i.e., with the flat side contacting the loading nose). Maximum flexural stress of each specimen was calculated using Equation (1), where P is the applied load, L the span of the sample, $b$ the width of the sample, and $h$ the thickness.

$$
\sigma=\frac{3 P L}{2 b h^{2}}
$$

Density tests: After flexural testing of the specimens, one section of each (approximately $50 \%$ of the total sample mass) was used to determine the average material density. Tests were conducted per sample using a Qualitest ${ }^{\mathrm{TM}}$ MDS-300 dens meter.

\section{Results and discussion}

\section{Observational results}

After 270 days of environmental exposure on the weathering station, samples underwent several physical changes including discoloration on the flat-mould exposed surface, gaining a cloudy appearance within the resin matrix (Figure 2). Extreme bowing in the resin samples in the upwards direction as shown in Figure 3 became more pronounced over time. It is believed that the resin absorbed a significant amount of moisture, causing volumetric changes, ${ }^{4}$ yet the boundary conditions of the clamp design along with a gradient of material properties on the two sides of the sample-note that the upper face would be exposed to UV more-have caused the plate expansion to result in an asymmetrical bowing. The porosity in most samples also became visually pronounced over time (Figure 4). Density measurements were taken before and after exposure of the laminates, but the uptake of water over time made it impossible to attribute changes in density due to moisture or slight dimensional changes as voids formed. Yet, it is believed that the latter mechanism is unlikely.

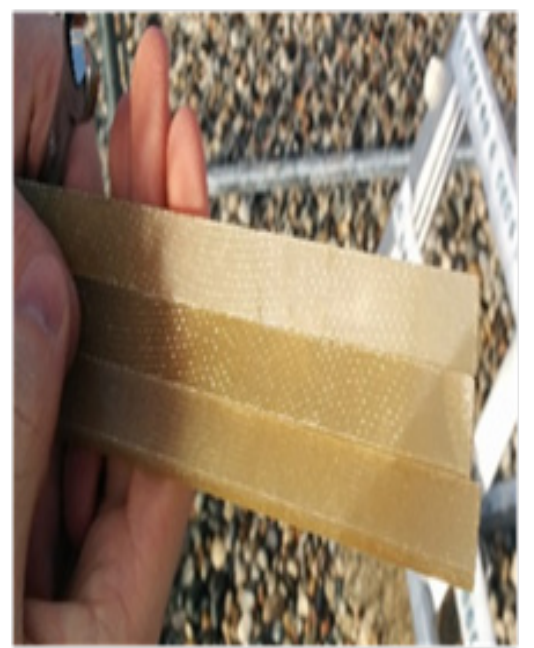

Figure 2 Discoloration of samples after 270 days of environmental exposure, on both the tool and open manufactured side of the specimens.

\section{Statistical analysis}

As described in Section 2.1, three factors of the analysis included:

a. Cure: The type of curing procedure used (either oven or ambient)

b. Material: The type of fibre preform architecture (random chopped or woven)

c. Coating: The application or no-application of gel coat 


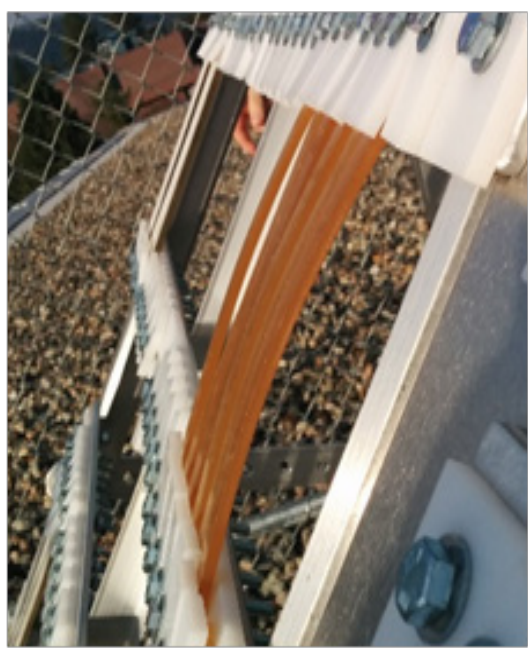

Figure 3 Bowing after 270 days of environmental exposure, for pure resin samples on the exposure rack.

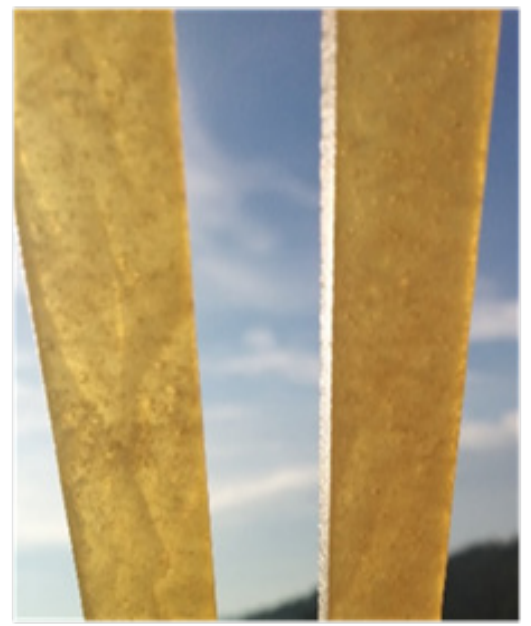

Figure 4 Persistent porosity in samples seen after 270 days of environmental exposure.

In order to determine the effects of these material- and processrelated parameters, ANOVA analysis was conducted using IBM SPSS Statistical package on the measured material properties (flexural stress, surface roughness, surface hardness and density) of both environmentally exposed samples and non-environmentally exposed(control) samples, in two separate blocks of time. Time was blocked in terms of the initial day of fabrication and after 270 days of exposure to the environmental conditions. The statistical analysis concluded only two significant factors (material and coating) having an effect on the dependent variables with a $p$-value $<5 \%$, in both exposure conditions. The degree of cure had no significant effect on the dependent variables, perhaps given its narrow range of variation (0.84-0.96) and effect on modulus development when compared to other two variables. Section 3.2.1 summarizes the statistical analysis for the samples group that received no environmental exposure, and section 3.2.2 summarizes the results for samples group with 270 days of environmental exposure.

No exposure-control group: Table 1 summarizes the ANOVA results under this group where the most significant factor influencing the hardness, strength and specific gravity of the parts was found to be the fibre preform type; the presence of gel coat did also have some significance on the part hardness and flexural strength, though with a lesser degree than fiber material. Given that no exposure was performed on this group of samples, these statistical results primarily indicated whether the sample fabrication and analysis routine were valid. We expect that in the case of maximum flexural stress, for example, that the fibre preform chosen would have a very significant effect, as laminates with continuous fibres are often much stronger than those with short fibres..$^{5}$ Density and hardness would also be affected by the choice of fibre preform, affecting the fibre volume fraction and other subsequent properties. Likewise, the addition of gel coat would slightly affect the maximum flexural stress, as varying the bulk laminate thickness alters the second moment of inertia. These expected relationships are present in Table 1 and have lent confidence to the use of the same statistical analysis for the environmentally exposed group of sample.

Environmentally exposed group: Table 2 summarizes the statistical results, where fibre preform continues to remain the most significant factor influencing the strength and specific gravity, even after the 270 days of environmental exposure. This result indicates that although there is likely a degradation of laminate properties from exposure, the rate of degradation between the two fibre preform choices is fairly consistent, keeping the two highly distinguishable from each other. Yet, in support of literature indicating that continuous fibres may draw moisture into the laminate more readily and hastening degradation, it can be observed that in the control samples (Figure 5), the average flexural strength of the continuous fibre laminates is approximately $67 \%$ higher than that of the chopped random fibre laminates. After the exposure time, the continuous fibre laminates (Figure 6) are approximately $47 \%$ higher in their flexural strength. This increased rate of degradation could be due to the aforementioned hypothesised mechanism of capillary action by the continuous fibres. ${ }^{4}$ Additionally, after the 270 days of exposure, fibre preform has become an insignificant factor with respect to hardness, as opposed to the control samples. Given the nature of the Shore D hardness test, being highly localised in its measurement, this change could be attributed to an increased sensitivity to resin properties in the local region, rather than material stiffness related to fibres close to the measurement surface.

Comparing Tables $1 \&$ Table 2, the presence of gel coat gained some significance over time in regards to flexural strength and roughness of the environmentally exposed specimens. This was expected, as gel coats are used on GFRP laminates to increase their environmental durability, especially in the areas of moisture ingress which would hasten the degradation of the polymer and hence flexural strength and surface quality/appearance. ${ }^{5}$ The impact of gel coat on density of the specimens after exposure is still appreciable, supporting the conclusion that the gel coat is performing its expected function.

\section{Making decisions on performance of GFRPs with environmental effects: an example}

It is no secret that the effects of initial material selection and manufacturing decisions have a significant impact on the desired outcome of a finished composite product. A manufacturer may undoubtedly choose a more cost and time effective laminate design and process to create the desired material form, especially in specific industrial contexts where standard design specifications are not well established. This article as part of a longer term research program was aimed to assist manufactures of GFRPs on such choices in the context of whether or not part longevity under natural environmental exposure would be of concern. In order to exemplify comparison framework 
based on the obtained results, let us consider a decision-making scenario for a manufacturer as follows; the design and processing factors in a base case include the use of a random chopped fibre mat material, unmonitored ambient cure conditions, and no application of gel coat i.e., Case 1 as shown in Table 3 . If the manufacturer is to alter the design and fabrication conditions to include a woven fibre material, controlled high degree of cure conditions and the application of gel coat (Case 2), we can predict the effect of these decisions on the performance of the laminate, depending on whether the part will be used in indoor room condition (Table 4) or in outdoors (Table 5). An example of this, using the obtained experimental results, is that although it is well established that the choice of a continuous woven reinforcement will provide a stronger laminate for tensile and flexural loading in nominal conditions, this is very much subject to change over time under natural environmental conditions.

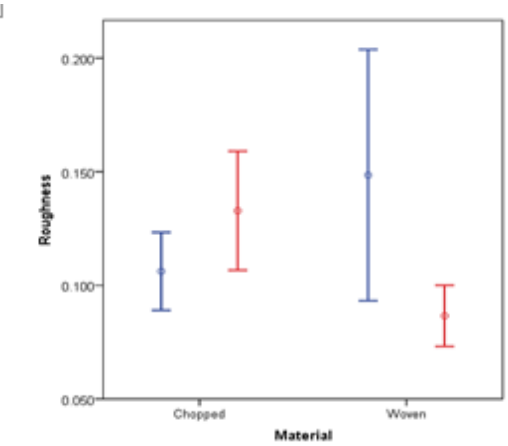

(A)

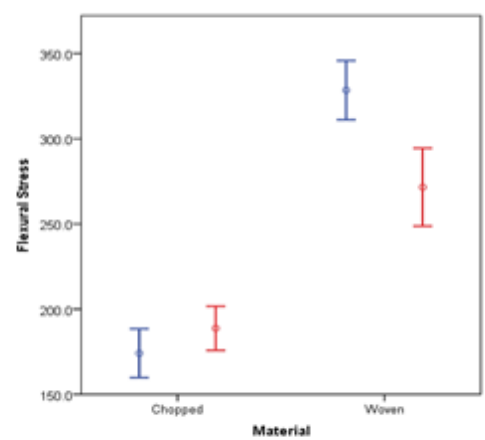

(C)

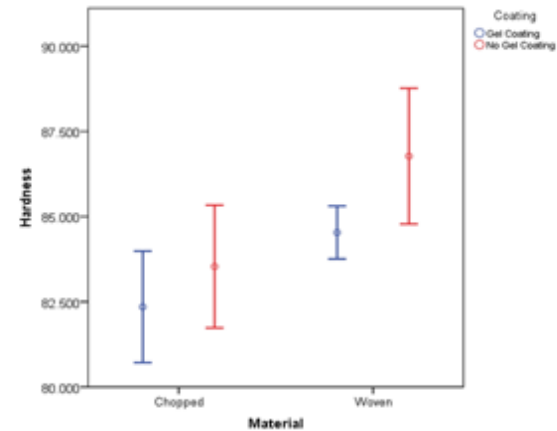

(B)

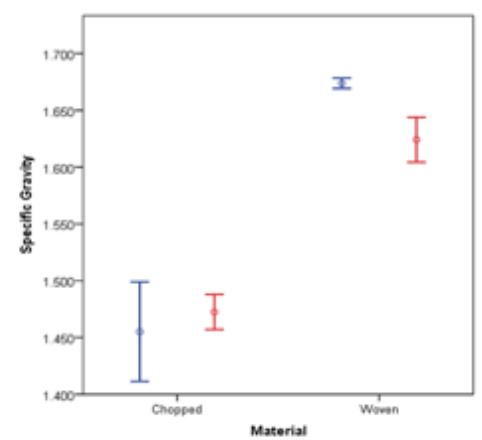

(D)

Figure 5 Material type and coating application factor plots for the four measured outputs, on samples with noenvironmental exposure; (A) surface roughness $\left(R_{a}-\mu m\right)$, (B) Shore (D) Hardness, (C) maximum flexural stress (MPa), (D) specific gravity (-).

Table I Statistical analysis results of non-environmentally exposed samples $(\alpha=0.05)$

\begin{tabular}{|c|c|c|c|c|c|c|c|}
\hline Source & & $\begin{array}{l}\text { Sum of } \\
\text { squares }\end{array}$ & df & Mean square & $\mathbf{F}$ & P-value & $\begin{array}{l}\text { Relative } \\
\text { significance }\end{array}$ \\
\hline \multirow{4}{*}{ Material } & Roughness & 2.6IE-05 & I & 2.6IE-05 & 0.055 & 0.818 & Insignificant \\
\hline & Hardness & 37.632 & I & 37.632 & 23.101 & 0 & $\sqrt{ }$ High \\
\hline & Flexural_Stress & 68661.786 & I & 68661.786 & 382.423 & 0 & $\sqrt{ }$ Very High \\
\hline & Specific_Gravity & 0.156 & I & 0.156 & 230.749 & 0 & $\sqrt{ }$ Very High \\
\hline \multirow{4}{*}{ Coating } & Roughness & 0.002 & I & 0.002 & 3.268 & 0.092 & Insignificant \\
\hline & Hardness & 15.552 & I & 15.552 & 9.547 & 0.008 & $\sqrt{ }$ Moderate \\
\hline & Flexural_Stress & 1943.898 & I & 1943.898 & 10.827 & 0.005 & $\sqrt{ }$ Moderate \\
\hline & Specific_Gravity & 0.002 & I & 0.002 & 3.129 & 0.099 & Insignificant \\
\hline \multirow{4}{*}{ Error } & Roughness & 0.007 & 14 & 0 & - & - & - \\
\hline & Hardness & 22.807 & 14 & 1.629 & - & - & - \\
\hline & Flexural_Stress & 2513.615 & 14 & 179.544 & - & - & - \\
\hline & Specific_Gravity & 0.009 & 14 & 0.001 & - & - & - \\
\hline
\end{tabular}




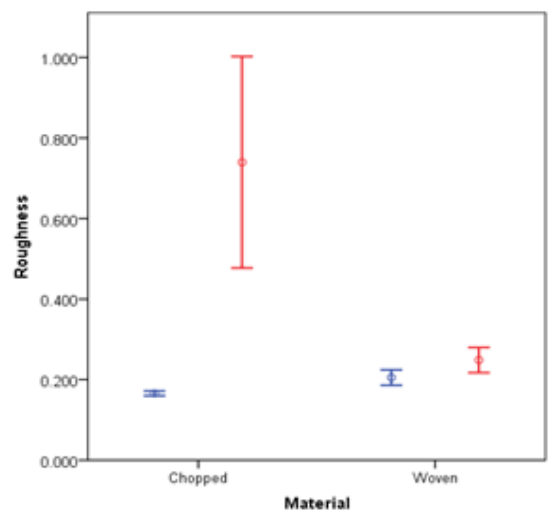

(A)

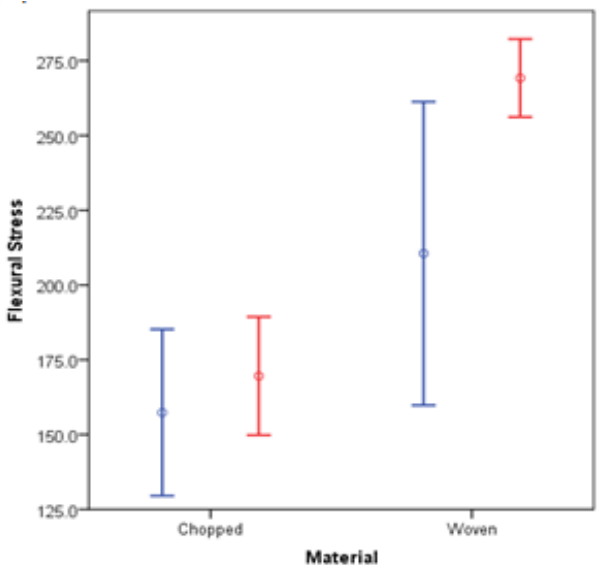

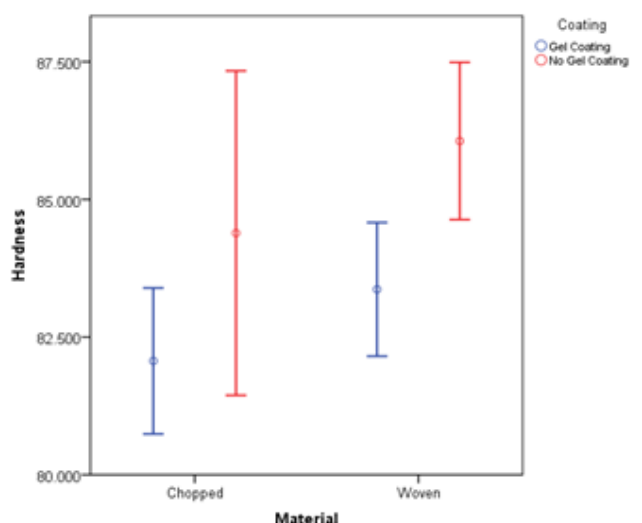

(B)

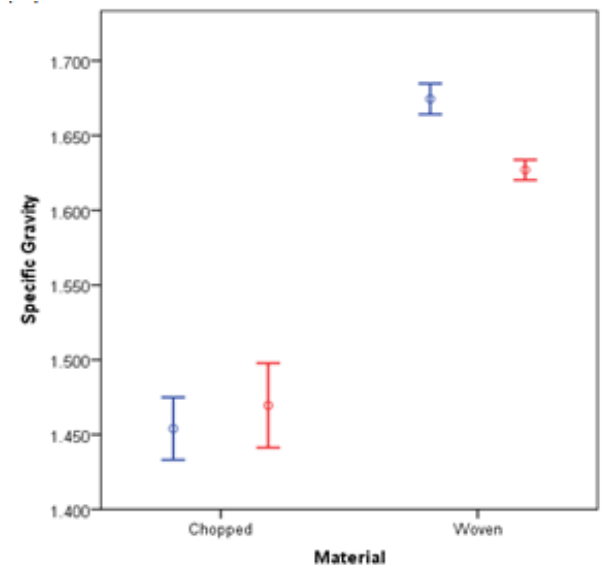

(D)

Figure 6 Effects plot for the four measured outputs on the samples after 270 days of environmental exposure; (A) surface roughness ( $\left.R_{a}-\mu m\right)$, (B) Shore (D) Hardness, (C) maximum flexural stress (MPa), (D) specific gravity (-).

Table 2 Statistical analysis results of 270 day exposed samples $(\alpha=0.05)$

\begin{tabular}{|c|c|c|c|c|c|c|c|}
\hline Source & & $\begin{array}{l}\text { Sum of } \\
\text { squares }\end{array}$ & df & Mean square & $\mathbf{F}$ & P-value & $\begin{array}{l}\text { Relative } \\
\text { significance }\end{array}$ \\
\hline \multirow{4}{*}{ Material } & Roughness & 0.27 & I & 0.27 & 9.369 & 0.016 & VLow \\
\hline & Hardness & 8.506 & I & 8.506 & 2.094 & 0.186 & Insignificant \\
\hline & Flexural_Stress & 23350.285 & I & 23350.285 & 98.118 & 0 & VVery High \\
\hline & Specific_Gravity & 0.143 & I & 0.143 & 840.494 & 0 & $\sqrt{ }$ Very High \\
\hline \multirow{4}{*}{ Coating } & Roughness & 0.462 & I & 0.462 & 15.998 & 0.004 & $\sqrt{ }$ High \\
\hline & Hardness & 37.003 & I & 37.003 & 9.11 & 0.017 & VLow \\
\hline & Flexural_Stress & 5022.982 & I & 5022.982 & 21.107 & 0.002 & $\sqrt{ }$ High \\
\hline & Specific_Gravity & 0.001 & I & 0.001 & 6.024 & 0.04 & VLow \\
\hline \multirow{4}{*}{ Error } & Roughness & 0.231 & 8 & 0.029 & - & - & - \\
\hline & Hardness & 32.496 & 8 & 4.062 & - & - & - \\
\hline & Flexural_Stress & 1903.852 & 8 & $237.98 I$ & - & - & - \\
\hline & Specific_Gravity & 0.001 & 8 & 0 & - & - & - \\
\hline
\end{tabular}


Table 3 Figurative case studies and the associated design and processing decisions

\begin{tabular}{llll}
\hline & Material & Cure & Coating \\
\hline Case I & Random chopped fibre & Ambient cure & No gel coat applied \\
Case 2 & Woven fibre & Oven cure & Gel coat applied \\
\hline
\end{tabular}

Table 4 Statistical differences between cases I and with no environmental exposure (indoor application of the part)

\begin{tabular}{lllll}
\hline & Roughness & Hardness & Flexural strength & Specific gravity \\
\hline $\begin{array}{l}\text { Material } \\
\text { (From Chopped to Woven) }\end{array}$ & Insignificant & Significant $\uparrow$ & Significant $\uparrow$ & Significant $\uparrow$ \\
$\begin{array}{l}\text { Cure } \\
\text { (From Room to Oven) }\end{array}$ & Insignificant & Insignificant & Insignificant & Insignificant \\
$\begin{array}{l}\text { Coating } \\
\text { (From no Gel to with Gel) }\end{array}$ & Insignificant & Significant $\downarrow$ & Significant $\uparrow$ & Insignificant \\
\hline
\end{tabular}

Table 5 Statistical differences between cases I and 2 after 270 days of environmental exposure (outdoor application of the part)

\begin{tabular}{lllll}
\hline & Roughness & Hardness & Flexural strength & Specific gravity \\
\hline $\begin{array}{l}\text { Material } \\
\text { (From Chopped to Woven) }\end{array}$ & Significant $\downarrow$ & Insignificant & Significant $\uparrow$ & Significant $\uparrow$ \\
$\begin{array}{l}\text { Cure } \\
\text { (From Room to Oven) }\end{array}$ & Insignificant & Insignificant & Insignificant & Insignificant \\
$\begin{array}{l}\text { Coating } \\
\text { (From No Gel to With Gel) }\end{array}$ & Significant $\downarrow$ & Significant $\downarrow$ & Significant $\downarrow$ & Significant $\uparrow$
\end{tabular}

For the non-exposed control samples, both gel coated and non gel coated, the average flexural stress for the chopped short fibre samples was $182 \mathrm{MPa}$ and for the continuous plain woven samples, was 297MPa. This comparison is very much in line with the aforementioned expectations. However, after 270 days of exposure, the average flexural stress for the chopped short fibre samples was $163 \mathrm{MPa}$ and for the continuous plain woven samples, was 232MPa. It has been noted that although the continuous fibre samples were still on average stronger than the short fibre samples, the former had a $21.9 \%$ reduction in strength over a 270-day exposure, whereas the latter had its strength reduced by $10.4 \%$. This stark difference can be attributed to the capillary action of continuous fibres, hastening the ingress of moisture inside the laminate and subsequent mechanical degradation, as also illustrated by the work of. ${ }^{4}$ This change would be expected to increase for periods greater than 270 days, as was investigated in this research program, illustrating that in some contexts of long-term exposure, the continuous fibre preform may or may not necessarily be a better engineering design choice.

Table 4 indicates the increasing/decreasing statistical trends when the fabrication is altered from Case 1 to Case 2 for the case of no exposure to natural environmental conditions. It can be seen that in the control group tested within days of fabrication, the woven long fibre architecture had a significant effect on the hardness, flexural strength and specific gravity of the laminate. The application of a gel coat had a tendency to significantly decrease the hardness of the part, whereas the application of an oven cure (at 50C) had statistically no significant effect on these outputs within the tested temperature range.

Similarly, Table 5 indicates the statistical relationships between the design and process choices of Case 1 to Case 2, with specimens exposed to natural environmental conditions for 270 days (i.e., assuming outdoor application of the part under design). After 270 days of environmental exposure, the choice of using continuous woven fibres in the laminate has a significant impact on the laminate surface roughness, by decreasing the degradation in surface quality over time, as well increase in the flexural strength and specific gravity when compared to choosing chopped random fibre mat as the reinforcement. The cure application continues to have no significant effect on the physical and mechanical property outputs, which was originally unexpected, as it is known that degree of cure has an impact on the uptake of moisture. ${ }^{6}$ Again, a reason for this observation can be due to limited change of cure degree between the two tested corresponding levels, mostly attributed to the slow cure kinetics and modulus development of the type of resin used above glass transition temperature. ${ }^{7}$ The application of a gel coating, after 270 days exposure has provided a statistically significant effect on all physical and mechanical properties in Table 5.

When comparing Tables $4 \&$ Table 5 , it is clear that material and process decisions make more number of significant effects (shown in red) when the long-term environmental performance of the part is concerned. Another very interesting observation is that the gel coating over exposure time has caused a negative significant effect on the composite flexural strength, specifically for the long-fiber woven composite also compare (Figures 5 \& 6).

\section{Conclusion}

Polymer matrix composites, such as glass-fibre reinforced polymers (GFRPs), are known to degrade over time when exposed to natural environmental conditions. Types of degradation can include reduced strength, lower surface quality and changes in density, among others. By exposure to ultraviolet radiation, moisture and temperature fluctuations, physical and mechanical properties of composites are subject to different degradation trends, depending on the initial design choices and properties of the fibre/resin materials. There is currently limited information and guidelines available to help designers and 
manufacturers better produce GFRP parts with an understanding of product longevity under different design and manufacturing conditions. This preliminarily study shows that the significance level of the effect of fiber architecture (long fiber vs short fiber), cure condition, and gel coat application on mechanical and physical properties of GFRPs can notably alter over time under natural weathering conditions. Hence, initial design and manufacturing choices should be made based on environmental exposure conditions of the part over it service life.

\section{Acknowledgements}

The authors would like to acknowledge financial support from the Natural Sciences and Engineering Research Council of Canada, as well as the UBC Work-Study Program. The work was also presented at the Canadian - International Conference on Composites (CANCOM15), August 18-20, Edmonton, Canada.

\section{Conflict of interest}

The author declares no conflict of interest.

\section{References}

1. Wang Y, Meng J, Zhao Q, et al. Accelerated ageing tests for evaluations of a durability performance of glass-fibre reinforcement polyester composites. Journal of Materials Science \& Technology. 2010;26(6):572576.
2. Abeysinghe HP, Edwards W, Pritchard G, et al. Degradation of cross linked resins in water and electrolyte solutions. Polymer. 1982;23(12):1785-1790.

3. Mouzakis DE, Zoga H, Galiotis C. Accelerated environmental ageing study of polyester/glass fibre reinforced composites (GFRPCs). Composites Part B. 2008;39(3):467-475.

4. Yunn Tzu Yu, Kishore P. Modeling long-term degradation due to moisture and oxygen in polymeric matrix composites. Materials Science and Engineering A. 2008;498(1-2):162-165.

5. Strong BA. Fundamentals of Composites Manufacturing: Materials, Methods and Applications. Dearborn, USA: Society of Manufacturing Engineers; 2008.

6. Guidelines for Characterization of Structural Materials, $\mathrm{CMH}-17$ Composite Materials Handbook. Volume 1-Revision G, Chapter 2, Wichita, USA: SAE International; 2012.

7. Mohammadi MS, Solnickova L, Crawford B, et al. Investigating the unrecovered displacement of glass fibre reinforced polymers due to manufacturing conditions. Journal of Multifunctional Composites. 2014;2(2):100-104. 